\title{
Optimizing depth of sedation for colonoscopy
}

\author{
Matthew T. V. Chan, MBBS, PhD • William K. K. Wu, PhD • Raymond S. Y. Tang, MD
}

Received: 29 July 2015/ Accepted: 13 August 2015/Published online: 26 August 2015

(c) Canadian Anesthesiologists' Society 2015

Colonoscopy is one of the most common investigative procedures performed in contemporary medicine. With the promulgation of national guidelines for the early detection of colorectal cancer, ${ }^{1}$ it is estimated that millions of colonoscopies are performed worldwide and the demand is likely to grow further each year. Despite the introduction of smaller and more flexible endoscopes, colonoscopy is still associated with significant pain and discomfort. Sedation is therefore generally required for the procedure. Given the large volume of service required, healthcare providers are constantly looking for ways to improve efficiency. In some countries, nurses are trained to administer sedation, ${ }^{2}$ others have used computer feedback systems to provide sedation during colonoscopy. ${ }^{3,4}$ Typically, these techniques are limited to provide light sedation that may minimize hemodynamic changes, allow faster emergence, and expedite patient turnover. Nevertheless, patients may become agitated and awake during the procedure, resulting in serious complications. There has yet to be a consensus among endoscopists, anesthesiologists, and

M. T. V. Chan, MBBS, PhD (凶) · W. K. K. Wu, PhD

Department of Anaesthesia and Intensive Care, The Chinese University of Hong Kong, Hong Kong Special Administrative Region, China

e-mail: mtvchan@cuhk.edu.hk

W. K. K. Wu, PhD - R. S. Y. Tang, MD

State Key Laboratory of Digestive Disease, Department of Medicine \& Therapeutics and LKS Institute of Health Sciences, The Chinese University of Hong Kong, Hong Kong Special Administrative Region, China

R. S. Y. Tang, MD

Institute of Digestive Disease, The Chinese University of Hong

Kong, Hong Kong Special Administrative Region, China patients as to whether deep or light sedation should be provided for colonoscopy.

In the current edition of the Journal, Allen et al. report a randomized equivalence trial comparing deep $v s$ light sedation using target-controlled propofol infusion in 199 patients having outpatient colonoscopy. ${ }^{5}$ Depth of sedation was measured using the bispectral electroencephalographic (EEG) index (i.e., bispectral index [BIS]) aimed at a BIS value of 70-80 or $<60$ for "light" and "deep" sedation, respectively. The primary outcome was unintentional recall of the procedure. In addition, hemodynamic, respiratory, and airway complications were carefully recorded. The patients, anesthesiologists, and endoscopists reported their level of satisfaction with sedation, and there was also a detailed assessment of cognitive function at discharge from the endoscopy unit. Not surprisingly, larger doses of sedatives were given for deep anesthesia. In patients receiving deep sedation, there was a lower rate of procedural recall $(1.0 \% \mathrm{vs}$ $12.9 \%$, deep $v s$ light sedation, respectively; $P=0.001$ ) and fewer episodes of patient movements during the procedure (11\% vs 22\%, respectively; $P=0.033$ ). Hypotension was more common with deep sedation than with light sedation (46\% vs 24\%, respectively; $P=0.001$ ), though episodes that required interventions with fluid or vasopressor administration were comparable between groups (14\% vs $9 \% ; P=0.279)$. Interestingly, patients did not seem to be bothered by the procedural recall, and virtually all patients (deep sedation 99\% vs light sedation 97\%; $P=0.369$ ) reported high satisfaction with the sedation they received. Cognitive performance at hospital discharge was also similar between groups.

There are safety concerns with deep sedation. ${ }^{6}$ As patient responses are blunted by large doses of hypnotic and analgesic agents, it is commonly viewed that deep sedation may increase the risk of aspiration and 
Table Airway and hemodynamic adverse events during sedation for gastrointestinal endoscopy

\begin{tabular}{|c|c|c|c|c|c|c|c|}
\hline \multirow[t]{2}{*}{ Author, year } & \multirow[t]{2}{*}{ Country } & \multirow[t]{2}{*}{ Study design } & \multirow[t]{2}{*}{ Sedation techniques } & \multirow[t]{2}{*}{$\begin{array}{l}\text { Endoscopic } \\
\text { procedures }\end{array}$} & \multirow[t]{2}{*}{$\begin{array}{l}\text { No. of } \\
\text { patients }\end{array}$} & \multicolumn{2}{|c|}{$\begin{array}{l}\text { Sedation-related adverse events } \\
n(\%)\end{array}$} \\
\hline & & & & & & Airway* & Hemodynamic $\dagger$ \\
\hline $\begin{array}{l}\text { Clarke } \text { et al., } \\
\quad 2002^{10}\end{array}$ & Australia & Database review & $\begin{array}{l}\text { Non-anesthesiologists using } \\
\text { combination of propofol, } \\
\text { fentanyl and midazolam }\end{array}$ & $\begin{array}{l}\text { All GI } \\
\text { endoscopy }\end{array}$ & 28,472 & $107(0.38 \%)$ & $77(0.27 \%)$ \\
\hline $\begin{array}{l}\text { Rex, et al. } \\
\quad 2002^{11}\end{array}$ & USA & $\begin{array}{l}\text { Prospective data } \\
\text { collection }\end{array}$ & $\begin{array}{l}\text { Nurse administered propofol } \\
\text { sedation }\end{array}$ & $\begin{array}{l}\text { All GI } \\
\text { endoscopy }\end{array}$ & 2,222 & $5(0.23 \%)$ & $1(0.05 \%)$ \\
\hline $\begin{array}{l}\text { Walker et al., } \\
\quad 2003^{12}\end{array}$ & USA & $\begin{array}{l}\text { Prospective data } \\
\text { collection }\end{array}$ & $\begin{array}{l}\text { Nurse administered propofol } \\
\text { sedation }\end{array}$ & $\begin{array}{l}\text { All GI } \\
\text { endoscopy }\end{array}$ & 9,152 & $7(0.08 \%)$ & \\
\hline $\begin{array}{l}\text { Rex et al. } \\
\quad 2005^{13}\end{array}$ & USA & $\begin{array}{l}\text { Prospective data } \\
\text { collection }\end{array}$ & $\begin{array}{l}\text { Nurse administered propofol } \\
\text { sedation }\end{array}$ & Colonoscopy & 17,527 & $7(0.04 \%)$ & \\
\hline \multirow[t]{2}{*}{$\begin{array}{l}\text { Tohda et al., } \\
2006^{14}\end{array}$} & Japan & $\begin{array}{l}\text { Prospective data } \\
\text { collection }\end{array}$ & $\begin{array}{l}\text { Nurse administered propofol } \\
\text { sedation }\end{array}$ & $\begin{array}{l}\text { All GI } \\
\text { endoscopy }\end{array}$ & 27,500 & $1,842(6.70 \%)$ & $511(1.86 \%)$ \\
\hline & & & & $\begin{array}{l}\text { Colonoscopy } \\
\text { alone }\end{array}$ & 7,900 & $567(7.18 \%)$ & $276(3.49 \%)$ \\
\hline $\begin{array}{l}\text { Vargo et al. } \\
2006^{20}\end{array}$ & USA & Physician survey & $\begin{array}{l}\text { Endoscopist or anesthesiologist } \\
\text { administered propofol sedation }\end{array}$ & Colonoscopy & 11,683 & $77(0.66 \%)$ & $81(0.69 \%)$ \\
\hline $\begin{array}{l}\text { Külling et al., } \\
\quad 2007^{16}\end{array}$ & Switzerland & $\begin{array}{l}\text { Prospective data } \\
\text { collection }\end{array}$ & $\begin{array}{l}\text { Nurse administered propofol } \\
\text { sedation }\end{array}$ & $\begin{array}{l}\text { All GI } \\
\text { endoscopy }\end{array}$ & 27,061 & $629(2.32 \%)$ & \\
\hline \multirow[t]{2}{*}{$\begin{array}{l}\text { Sharma et al., } \\
2007^{17}\end{array}$} & USA & $\begin{array}{l}\text { Prospective data } \\
\text { collection }\end{array}$ & $\begin{array}{l}\text { General anesthesia or "conscious } \\
\text { sedation", unspecified regimen }\end{array}$ & $\begin{array}{l}\text { All GI } \\
\text { endoscopy }\end{array}$ & 324,737 & $1,049(0.32 \%)$ & $2,244(0.69 \%)$ \\
\hline & & & & $\begin{array}{l}\text { Colonoscopy } \\
\text { alone }\end{array}$ & 179,521 & $565(0.31 \%)$ & $1,805(1.01 \%)$ \\
\hline \multirow[t]{2}{*}{$\begin{array}{l}\text { Jensen } \text { et al., } \\
2011^{18}\end{array}$} & Denmark & $\begin{array}{l}\text { Prospective data } \\
\text { collection }\end{array}$ & $\begin{array}{l}\text { Nurse administered propofol } \\
\text { sedation }\end{array}$ & $\begin{array}{l}\text { All GI } \\
\text { endoscopy }\end{array}$ & 1,822 & $78(4.28 \%)$ & \\
\hline & & & & $\begin{array}{l}\text { Colonoscopy } \\
\text { alone }\end{array}$ & 707 & $16(2.26 \%)$ & \\
\hline $\begin{array}{l}\text { Cooper et al., } \\
2013^{7}\end{array}$ & USA & Database review & $\begin{array}{l}\text { Sedation provided with and } \\
\text { without anesthesia assistance } \\
\text { (unspecified regimen) }\end{array}$ & Colonoscopy & 165,527 & $125(0.10 \%)$ & $48(0.14 \%)$ \\
\hline $\begin{array}{l}\text { Sieg et al., } \\
\qquad 2014^{19}\end{array}$ & Germany & $\begin{array}{l}\text { Prospective data } \\
\text { collection }\end{array}$ & $\begin{array}{l}\text { Endoscopist administered propofol } \\
\text { sedation }\end{array}$ & $\begin{array}{l}\text { All GI } \\
\text { endoscopy }\end{array}$ & 24,441 & $97(0.40 \%)$ & $12(0.05 \%)$ \\
\hline \multirow[t]{2}{*}{$\begin{array}{l}\text { Jensen et al., } \\
2015^{9}\end{array}$} & Denmark & Database review & $\begin{array}{l}\text { Nurse administered propofol } \\
\text { sedation }\end{array}$ & $\begin{array}{l}\text { All GI } \\
\text { endoscopy }\end{array}$ & 6,840 & $214(3.13 \%)$ & $204(2.98 \%)$ \\
\hline & & & & $\begin{array}{l}\text { Colonoscopy } \\
\text { alone }\end{array}$ & 4,347 & $130(2.99 \%)$ & $154(3.54 \%)$ \\
\hline
\end{tabular}

GI = gastrointestinal; *Airway events include partial or complete obstruction of airway, apnea, and hypoxia. $\dagger$ Hemodynamic changes include hypotension, arrhythmia, tachy- or bradycardia

hemodynamic instability. In a database review of 165,527 patients having colonoscopy, deep sedation - as defined by the administration of propofol-increased the risk of aspiration, inadvertent splenic injury, or colonic perforation when compared with light sedation without the use of propofol (odds ratio, 1.46; 95\% confidence interval, 1.09 to $1.94 ; P<0.001) .^{7}$ Nevertheless, the observations could not be reproduced by other investigators. $^{8,9}$ It should be pointed out that none of these studies actually measured depth of sedation, either clinically or based on EEG. The results were also limited by the inherent risk of retrospective analysis with residual confounding, missing data, and potential misclassification of cases having deep vs light sedation. As a randomized trial, Allen's study is therefore unique and important because it produced unbiased data with a head-to-head comparison between deep and light sedation.

Nevertheless, interpretation of Allen's study does require considerable caution. The Table shows the rates of adverse events following sedation for endoscopy in studies with at least a thousand patients. ${ }^{7,9-19}$ In 646,984 patients, the average [interquartile range] rates of airway and hemodynamic events were $1.44 \%$ [0.05-2.93] and $0.74 \%$ [0.04-1.28], respectively. In contrast, irrespective of depth of sedation, more than $20 \%$ of patients in Allen's study had an airway event requiring one of the basic airway maneuvers. In $2 \%$ of patients, an oropharyngeal or nasopharyngeal airway was required to maintain airway 
patency. Similarly, $11 \%$ of patients received vasopressors or fluid boluses to correct hemodynamic concerns. Although underreporting in previous studies should be considered, variations in endpoint definitions will certainly contribute to the discrepancy in the rates of adverse events. These data argue strongly for standardized endpoint measures to define the magnitude of the problem and the predictors of adverse events and to explore potential interventions for better outcomes. In our view, based on the reported rate of adverse events in Allen's study, a dedicated practitioner skilled in airway and hemodynamic management should administer sedation for colonoscopy.

It should also be pointed out that the majority of the procedures in Allen's study were completed within $20 \mathrm{~min}$, suggesting that the bulk of the procedures were performed for diagnostic purposes where surgical stimuli are likely to be milder and constant throughout the examination. Thus, it remains unclear whether the results could be extrapolated to the more complex and lengthy therapeutic colonoscopy. Specifically, it is uncertain whether light sedation alone would be sufficient for the more complex therapeutic procedures such as endoscopic mucosal resection or endoscopic submucosal dissection for large polyps. One should be mindful that exaggerated responses during these interventional procedures may produce serious harmful effects. For instance, patient movement during resection of a large sessile polyp may obscure surgical exposure and increase the risk of surgical error, bleeding, and inadvertent perforation of the colon.

So, what is the optimal depth of sedation for colonoscopy? Given the high degree of patient satisfaction and faster emergence in the recovery room, it may be tempting to offer light sedation initially, especially when the patient is aware of and prepared to accept the risk of recall during the procedure. Then again, it is equally important to be prepared for situations that urgently require deep sedation and to be able to recognize and act on changes in airway and hemodynamics in a timely fashion.

\section{Optimiser la profondeur de la sédation pour la colonoscopie}

La colonoscopie est l'une des procédures diagnostiques le plus souvent pratiquées de la médecine contemporaine. Avec la publication des lignes directrices nationales pour la détection précoce du cancer colorectal, ${ }^{1}$ on estime que des millions de colonoscopies sont réalisées dans le monde et la demande est susceptible de crô̂tre chaque année. En dépit de l'introduction d'endoscopes plus petits et plus flexibles, la colonoscopie reste associée à une douleur et à une gêne significatives. C'est pourquoi une sédation est généralement requise pour la procédure. Considérant la grande quantité de services requis, les fournisseurs de soins de santé recherchent continuellement des moyens d'améliorer l'efficacité. Dans certains pays, des infirmières sont formées pour administrer la sédation; ${ }^{2}$ ailleurs, on a utilisé des systèmes de rétroaction informatisés pour assurer la sédation au cours de la colonoscopie. ${ }^{3,4}$ Ces techniques se limitent habituellement à une sédation légère qui peut minimiser les modifications hémodynamiques, permettre une émergence plus rapide et accélérer la rotation des patients. Des patients peuvent néanmoins devenir agités et s'éveiller au cours de la procédure, ce qui peut aboutir à de graves complications. Il reste encore à trouver un consensus entre les endoscopistes, les anesthésiologistes et les patients pour déterminer s'il faut procurer une sédation légère ou profonde pour une colonoscopie.

Dans ce numéro du Journal, Allen et coll. rapportent un essai randomisé d'équivalence comparant la sédation profonde à la sédation légère en utilisant une perfusion de propofol à concentration cible chez 199 patients ambulatoires subissant une colonoscopie. ${ }^{5}$ La profondeur de la sédation a été mesurée au moyen d'un indice encéphalographique (EEG) bispectral (c'est-à-dire, l'indice bispectral [BIS]) visant une valeur de 70 à 80 ou $<60$ pour, respectivement, une sédation «légère » ou une sédation «profonde ». Le critère d'évaluation principal était la mémoire involontaire de la procédure. De plus, les complications hémodynamiques, respiratoires et des voies aériennes ont été soigneusement consignées. Les patients, les anesthésiologistes et les endoscopistes ont communiqué leur niveau de satisfaction relativement à la sédation et le service d'endoscopie a réalisé une évaluation détaillée des fonctions cognitives au moment du congé du patient. Sans surprise, les doses de sédatifs les plus fortes ont été administrées pour une anesthésie profonde. Le taux de mémoire de la procédure a été moins élevé pour les patients recevant une sédation profonde que pour une sédation légère (respectivement, $1,0 \%$ contre $12,9 \% ; p=$ 0,001 ) et il y a eu moins d'épisodes de mouvements des patients en cours de procédure en cas de sédation profonde (respectivement, $11 \%$ contre $22 \% ; \quad P=0,033$ ). L'hypotension a été plus fréquente en cas de sédation profonde qu'en cas de sédation légère (respectivement, $46 \%$ contre $24 \% ; P=0,001)$, bien que le pourcentage d'épisodes ayant nécessité une intervention avec l'administration de liquides ou de vasopresseurs ait été comparable entre les deux groupes (14\% contre $9 \%$; $P=0,279)$. Il est intéressant de noter que les patients n'ont pas semblé gênés par la mémoire de la procédure et quasiment tous les patients (sédation profonde $99 \%$ contre 
Tableau Évènements indésirables des voies aériennes et hémodynamiques au cours de la sédation pour endoscopie digestive

\begin{tabular}{|c|c|c|c|c|c|c|c|}
\hline \multirow[t]{2}{*}{$\begin{array}{l}\text { Auteur, } \\
\text { année }\end{array}$} & \multirow[t]{2}{*}{ Pays } & \multirow[t]{2}{*}{$\begin{array}{l}\text { Schéma de } \\
\text { l'étude }\end{array}$} & \multirow[t]{2}{*}{ Techniques de sédation } & \multirow[t]{2}{*}{$\begin{array}{l}\text { Procédures } \\
\text { endoscopiques }\end{array}$} & \multirow{2}{*}{$\begin{array}{l}\text { Nombre } \\
\text { de } \\
\text { patients }\end{array}$} & \multicolumn{2}{|c|}{$\begin{array}{l}\text { Évènements indésirables liés à la } \\
\text { sédation } n(\%)\end{array}$} \\
\hline & & & & & & $\begin{array}{l}\text { Voies } \\
\text { aériennes* }\end{array}$ & Hémodynamique $\dagger$ \\
\hline $\begin{array}{l}\text { Clarke et } \\
\text { coll. }, \\
2002^{10}\end{array}$ & Australie & $\begin{array}{l}\text { Analyse d'une } \\
\text { base de } \\
\text { données }\end{array}$ & $\begin{array}{l}\text { Non-anesthésiologistes utilisant une } \\
\text { combinaison de propofol, fentanyl et } \\
\text { midazolam }\end{array}$ & $\begin{array}{l}\text { Toutes } \\
\text { endoscopies } \\
\text { digestives }\end{array}$ & 28472 & $107(0,38 \%)$ & $77(0,27 \%)$ \\
\hline $\begin{array}{l}\text { Rex, et } \\
\quad \text { coll. } \\
2002^{11}\end{array}$ & États-Unis & $\begin{array}{l}\text { Collecte } \\
\text { prospective } \\
\text { de données }\end{array}$ & $\begin{array}{l}\text { Sédation par propofol administré par } \\
\text { infirmier/ère }\end{array}$ & $\begin{array}{l}\text { Toutes } \\
\text { endoscopies } \\
\text { digestives }\end{array}$ & 2222 & $5(0,23 \%)$ & $1(0,05 \%)$ \\
\hline $\begin{array}{l}\text { Walker et } \\
\text { coll. } \\
2003^{12}\end{array}$ & États-Unis & $\begin{array}{l}\text { Collecte } \\
\text { prospective } \\
\text { de données }\end{array}$ & $\begin{array}{l}\text { Sédation par propofol administré par } \\
\text { infirmier/ère }\end{array}$ & $\begin{array}{l}\text { Toutes } \\
\text { endoscopies } \\
\text { digestives }\end{array}$ & 9152 & $7(0,08 \%)$ & \\
\hline $\begin{array}{l}\text { Rex et } \\
\quad \text { coll. } \\
2005^{13}\end{array}$ & États-Unis & $\begin{array}{l}\text { Collecte } \\
\text { prospective } \\
\text { de données }\end{array}$ & $\begin{array}{l}\text { Sédation par propofol administré par } \\
\text { infirmier/ère }\end{array}$ & Colonoscopie & 17527 & $7(0,04 \%)$ & \\
\hline \multirow[t]{2}{*}{$\begin{array}{l}\text { Tohda et } \\
\text { coll., } \\
2006^{14}\end{array}$} & Japon & $\begin{array}{l}\text { Collecte } \\
\text { prospective } \\
\text { de données }\end{array}$ & $\begin{array}{l}\text { Sédation par propofol administré par } \\
\text { infirmier/ère }\end{array}$ & $\begin{array}{l}\text { Toutes } \\
\text { endoscopies } \\
\text { digestives }\end{array}$ & 27500 & $\begin{array}{l}1842 \\
\quad(6,70 \%)\end{array}$ & $511(1,86 \%)$ \\
\hline & & & & $\begin{array}{l}\text { Colonoscopie } \\
\text { uniquement }\end{array}$ & 7900 & $567(7,18 \%)$ & $276(3,49 \%)$ \\
\hline $\begin{array}{l}\text { Vargo et } \\
\text { coll., } \\
2006^{20}\end{array}$ & États-Unis & $\begin{array}{l}\text { Enquête auprès } \\
\text { des } \\
\text { médecins }\end{array}$ & $\begin{array}{l}\text { Un endoscopiste ou un } \\
\text { anesthésiologiste a administré une } \\
\text { sédation par propofol }\end{array}$ & Colonoscopie & 11683 & $77(0,66 \%)$ & $81(0,69 \%)$ \\
\hline $\begin{array}{l}\text { Külling et } \\
\text { coll., } \\
2007^{16}\end{array}$ & Suisse & $\begin{array}{l}\text { Collecte } \\
\text { prospective } \\
\text { de données }\end{array}$ & $\begin{array}{l}\text { Sédation par propofol administré par } \\
\text { infirmier/ère }\end{array}$ & $\begin{array}{l}\text { Toutes } \\
\text { endoscopies } \\
\text { digestives }\end{array}$ & 27061 & $629(2,32 \%)$ & \\
\hline \multirow[t]{2}{*}{$\begin{array}{l}\text { Sharma et } \\
\text { coll. } \\
2007^{17}\end{array}$} & États-Unis & $\begin{array}{l}\text { Collecte } \\
\text { prospective } \\
\text { de données }\end{array}$ & $\begin{array}{l}\text { Anesthésie générale ou « sédation } \\
\text { consciente », protocole non précisé }\end{array}$ & $\begin{array}{l}\text { Toutes } \\
\text { endoscopies } \\
\text { digestives }\end{array}$ & 324737 & $\begin{array}{l}1049 \\
\quad(0,32 \%)\end{array}$ & $2244(0,69 \%)$ \\
\hline & & & & $\begin{array}{l}\text { Colonoscopie } \\
\text { uniquement }\end{array}$ & 179521 & $565(0,31 \%)$ & $1805(1,01 \%)$ \\
\hline \multirow[t]{2}{*}{$\begin{array}{l}\text { Jensen } \text { et } \\
\quad \text { coll., } \\
2011^{18}\end{array}$} & Danemark & $\begin{array}{l}\text { Collecte } \\
\text { prospective } \\
\text { de données }\end{array}$ & $\begin{array}{l}\text { Sédation par propofol administré par } \\
\text { infirmier/ère }\end{array}$ & $\begin{array}{l}\text { Toutes } \\
\text { endoscopies } \\
\text { digestives }\end{array}$ & 1822 & $78(4,28 \%)$ & \\
\hline & & & & $\begin{array}{l}\text { Colonoscopie } \\
\text { uniquement }\end{array}$ & 707 & $16(2,26 \%)$ & \\
\hline $\begin{array}{l}\text { Cooper et } \\
\text { coll. } \\
2013^{7}\end{array}$ & États-Unis & $\begin{array}{l}\text { Analyse d'une } \\
\text { base de } \\
\text { données }\end{array}$ & $\begin{array}{l}\text { Sédation fournie avec ou sans } \\
\text { assistance anesthésique (protocole } \\
\text { non précisé) }\end{array}$ & Colonoscopie & 165527 & $125(0,10 \%)$ & $48(0,14 \%)$ \\
\hline $\begin{array}{l}\text { Sieg et } \\
\quad \text { coll. } \\
2014^{19}\end{array}$ & Allemagne & $\begin{array}{l}\text { Collecte } \\
\text { prospective } \\
\text { de données }\end{array}$ & $\begin{array}{l}\text { Sédation par propofol administré par } \\
\text { endoscopiste }\end{array}$ & $\begin{array}{l}\text { Toutes } \\
\text { endoscopies } \\
\text { digestives }\end{array}$ & 24441 & $97(0,40 \%)$ & $12(0,05 \%)$ \\
\hline \multirow[t]{2}{*}{$\begin{array}{l}\text { Jensen } \text { et } \\
\text { coll., } \\
2015^{9}\end{array}$} & Danemark & $\begin{array}{l}\text { Analyse d'une } \\
\text { base de } \\
\text { données }\end{array}$ & $\begin{array}{l}\text { Sédation par propofol administré par } \\
\text { infirmier/ère }\end{array}$ & $\begin{array}{l}\text { Toutes } \\
\text { endoscopies } \\
\text { digestives }\end{array}$ & 6840 & $214(3,13 \%)$ & $204(2,98 \%)$ \\
\hline & & & & $\begin{array}{l}\text { Colonoscopie } \\
\text { uniquement }\end{array}$ & 4347 & $130(2,99 \%)$ & $154(3,54 \%)$ \\
\hline
\end{tabular}

* les évènements des voies aériennes incluent: obstruction partielle ou complète de la voie aérienne, apnée et hypoxie. $\dagger$ Les modifications hémodynamiques incluent: hypotension, arythmie, tachy- ou bradycardie

sédation légère $97 \% ; P=0,369)$ ont fait part de leur grande satisfaction avec la sédation reçue. Les performances cognitives au congé de l'hôpital étaient également comparables entre les groupes.
La sédation profonde soulève des questions quant à son innocuité. ${ }^{6}$ Dans la mesure où les réponses des patients sont diminuées par les fortes doses d'hypnotiques et d'analgésiques, on estime habituellement que la sédation 
profonde peut augmenter le risque d'aspiration et d'instabilité hémodynamique. L'analyse d'une base de données sur 165527 patients ayant eu une colonoscopie, la sédation profonde (définie par l'administration de propofol) a augmenté le risque d'aspiration, de lésion accidentelle de la rate ou de perforation du côlon par rapport à la sédation légère sans propofol (rapport de cotes, 1,46; IC à $95 \%$ : $1,09$ à 1,$94 ; P<0,001){ }^{7}$ Néanmoins, ces observations n'ont pas pu être reproduites par d'autres investigateurs. ${ }^{8,9}$ Il faut préciser qu'aucune de ces études n'a véritablement mesuré la profondeur de la sédation, soit cliniquement, soit en se basant sur un EEG. Les résultats ont été également limités par les risques inhérents à une analyse rétrospective avec des données confondantes résiduelles ou manquantes, et une erreur de classification toujours possible des cas entre sédation profonde et légère. L'étude d'Allen, randomisée, est donc unique et importante parce qu'elle a fourni des données non biaisées sur une comparaison bilatérale entre sédation profonde et sédation légère.

L'interprétation de l'étude d'Allen exige néanmoins une prudence considérable. Le tableau montre les taux d'évènements indésirables après sédation pour endoscopie dans des études ayant inclus au moins un millier de patients. ${ }^{7,9-19}$ Sur 646984 patients, les taux moyens [intervalles interquartiles] d'évènements concernant les voies aériennes et d'évènements hémodynamiques ont été, respectivement de $1,44 \%[0,05$ - 2,93] et $0,74 \%$ [0,04-1,28]. En revanche, et indépendamment de la profondeur de la sédation, plus de $20 \%$ des patients dans l'étude d'Allen ont présenté un évènement des voies aériennes nécessitant une manœuvre de base sur les voies aériennes. Chez $2 \%$ des patients, il a été nécessaire d'insérer une canule de Guedel ou une trompette nasale pour maintenir la perméabilité de la voie aérienne. De même, $11 \%$ des patients ont reçu des bolus de vasopresseurs ou de liquides pour corriger des problèmes hémodynamiques. Même si l'on envisage une sous-déclaration dans les études précédentes, des variations dans les définitions des critères d'évaluation contribuent certainement aux divergences constatées dans les taux d'évènements indésirables. Ces données militent fortement pour des mesures standardisées de critères d'évaluation définissent l'amplitude du problème et des éléments prédictifs des évènements indésirables, et pour explorer des interventions potentielles en vue de meilleurs aboutissements. D'après le taux d'évènements indésirables publiés dans l'étude d'Allen, il faut—selon nous-que la sédation pour colonoscopie soit administrée par un praticien dédié et compétent dans la gestion des voies aériennes et des problèmes hémodynamiques.

Il faut également souligner que la majorité des procédures de l'étude d'Allen étaient complétées en moins de 20 minutes, ce qui suggère que l'essentiel de ces procédures était réalisé dans un but diagnostique rendant les stimulations chirurgicales probablement moindres et constantes tout au long de l'examen. Ainsi, nous ne pouvons pas dire avec certitude si ces résultats peuvent être extrapolés aux colonoscopies à but thérapeutique, plus complexes et plus longues. Il n'est, notamment, pas certain qu'une sédation légère seule puisse suffire pour des procédures thérapeutiques plus complexes telles que des résections endoscopiques de muqueuse ou la dissection endoscopique sous-muqueuse pour l'ablation de gros polypes. On doit garder à l'esprit que des réponses exagérées au cours de ces procédures opératoires peuvent entraîner des conséquences dommageables graves. Ainsi, le mouvement d'un patient pendant la résection d'un grand polype sessile peut obscurcir l'exposition chirurgicale et augmenter le risque d'erreur chirurgicale, de saignement et de perforation accidentelle du côlon.

Dans ces conditions, quelle est la profondeur optimale de la sédation pour la colonoscopie? Compte tenu du haut degré de satisfaction du patient et de l'émergence plus rapide en salle de réveil, il peut être tentant de proposer initialement une sédation légère, en particulier quand le patient est informé et prêt à accepter le risque de mémoires en cours de procédure. De nouveau, il est également important d'être préparé à affronter des situations qui requièrent d'urgence une sédation profonde et d'être capable de reconnaître et d'agir sur les voies aériennes et l'hémodynamique en temps opportun.

Conflicts of interest None declared.

Conflits d'intérêts Aucun déclaré.

\section{References}

1. Telford JJ. Canadian guidelines for colorectal cancer screening. Can J Gastroenterol 2011; 25: 479-81.

2. Vargo JJ, Cohen LB, Rex DK, et al. Position statement: Nonanesthesiologist administration of propofol for GI endoscopy. Gastroenterology 2009; 137: 2161-7.

3. Goudra BG, Singh PM, Chandrasekhara V. SEDASYS ${ }^{\circledR}$, airway, oxygenation, and ventilation: anticipating and managing the challenges. Dig Dis Sci 2014; 59: 920-7.

4. Pambianco DJ, Vargo JJ, Pruitt RE, Hardi R, Martin JF. Computer-assisted personalized sedation for upper endoscopy and colonoscopy: a comparative, multicenter randomized study. Gastrointest Endosc 2011; 73: 765-72.

5. Allen M, Leslie K, Hebbard G, Jones I, Mettho T, Maruff P. A randomized controlled trial of light versus deep propofol sedation for elective outpatient colonoscopy: recall, procedural conditions, and recovery. Can J Anesth 2015: 62: this issue. DOI:10.1007/ s12630-015-0463-3

6. Rex DK. Effect of the Centers for Medicare \& Medicaid Services policy about deep sedation on use of propofol. Ann Intern Med 2011; 154: 622-6. 
7. Cooper GS, Kou TD, Rex DK. Complications following colonoscopy with anesthesia assistance: a population-based analysis. JAMA Intern Med 2013; 173: 551-6.

8. Lubarsky DA, Guercio JR, Hanna JW, et al. The impact of anesthesia providers on major morbidity following screening colonoscopies. J Multidiscip Healthc 2015; 8: 255-70.

9. Jensen JT, Moller A, Hornslet P, Konge L, Vilmann P. Moderate and deep nurse-administered propofol sedation is safe. Dan Med J 2015; 62: A5049.

10. Clarke AC, Chiragakis L, Hillman LC, Kaye GL. Sedation for endoscopy: the safe use of propofol by general practitioner sedationists. Med J Aust 2002; 176: 158-61.

11. Rex DK, Overley $C$, Kinser $K$, et al. Safety of propofol administered by registered nurses with gastroenterologist supervision in 2000 endoscopic cases. Am J Gastroenterol 2002; 97: 1159-63.

12. Walker JA, McIntyre RD, Schleinitz PF, et al. Nurse-administered propofol sedation without anesthesia specialists in 9152 endoscopic cases in an ambulatory surgery center. Am J Gastroenterol 2003; 98: 1744-50.

13. Rex DK, Heuss LT, Walker JA, Qi R. Trained registered nurses/ endoscopy teams can administer propofol safely for endoscopy. Gastroenterology 2005; 129: 1384-91.

14. Tohda G, Higashi S, Wakahara S, Morikawa M, Sakumoto H, Kane T. Propofol sedation during endoscopic procedures: safe and effective administration by registered nurses supervised by endoscopists. Endoscopy 2006; 38: 360-7.

15. Vargo JJ, Holub JL, Faigel DO, Lieberman DA, Eisen GM. Risk factors for cardiopulmonary events during propofol-mediated upper endoscopy and colonoscopy. Aliment Pharmacol Ther 2006; 24: 955-63.

16. Kulling D, Orlandi $M$, Inauen $W$. Propofol sedation during endoscopic procedures: how much staff and monitoring are necessary? Gastrointest Endosc 2007; 66: 443-9.

17. Sharma VK, Nguyen CC, Crowell MD, Lieberman DA, de Garmo $P$, Fleischer $D E$. A national study of cardiopulmonary unplanned events after GI endoscopy. Gastrointest Endosc 2007; 66: 27-34.

18. Jensen JT, Vilmann P, Horsted T, et al. Nurse-administered propofol sedation for endoscopy: a risk analysis during an implementation phase. Endoscopy 2011; 43: 716-22.

19. Sieg A, bng Study G, Beck S, et al. Safety analysis of endoscopistdirected propofol sedation: a prospective, national multicenter study of 24441 patients in German outpatient practices. J Gastroenterol Hepatol 2014; 29: 517-23.

20. Vargo JJ, Holub JL, Faigel DO, Lieberman DA, Eisen GM. Risk factors for cardiopulmonary events during propofol-mediated upper endoscopy and colonoscopy. Aliment Pharm Therap 2006; 24: $955-63$. 